\title{
Development of an Underground In-Situ Stress Monitoring System for Mining Safety Using Multi Sensor Cell and Wi-Fi Direct Technology
}

\author{
Hajime Ikeda ${ }^{1(\&)}$, Youhei Kawamura ${ }^{1}$, Hyongdoo Jang ${ }^{2}$, \\ Nur Ellisha Binti Mokhtar ${ }^{3}$, Jun Yokokura ${ }^{3}$, and Zedrick Paul L. Tungol ${ }^{1}$ \\ Graduate School of International Resource Sciences, Akita University, \\ Akita 010-8502, Japan ha2ikeda@gmail.com \\ Western Australian School of Mines, Curtin University, \\ Kalgoorlie, WA 6430, Australia \\ Faculty of International Resource Sciences, Akita University, Akita 010-8502, Japan
}

1

\begin{abstract}
The increasing global demand for minerals contributes to the necessity of mineral extraction at greater depths. However, the increase of rock in-situ stress with depth leads to higher risk and increasingly dangerous working conditions faced by mining workers. The presence of shafts, tunnels and other excavations necessary in mine expansions further increase the complexity of underground mines. This complexity of underground stress conditions increases the importance of monitoring and analysis of underground strata conditions, as early detection is crucial in the prevention of rock failure and the occurrence of fatal accidents. A better comprehension of the underground stress conditions in a mine is vital in considering mine design and supports that need to be installed. The development of an efficient monitoring system that can obtain and transmit data is necessary. This paper suggests the utilisation of a multi sensor cell that combines the functions of an accelerometer, gyroscope and a magnetometer, as well as strain gauge displacements to continuously measure the stress conditions of bedrock. The obtained data is then conveyed to the surface using a Wi-Fi Direct communication system and analysed to comprehend the changes in the underground stress conditions. The latter part of this paper also describes the experiments conducted to verify the ability of the proposed monitoring system.
\end{abstract}

Keywords: Monitoring system In-situ stress Wi-Fi Direct

\section{Introduction}

In underground conditions, bedrock is subjected to stresses resulting from the load of the overburden and tectonic movements. Understanding the stress and strain (in-situ stress and displacement) conditions of the rock is important in the design and excavation of underground mines. Although, recent years have seen great progress in numerical computational methods such as FEM which allows more accurate numerical

(C) Springer Nature Switzerland AG 2020

E. Topal (Ed.): MPES 2019, SSGG, pp. 236-244, 2020.

https://doi.org/10.1007/978-3-030-33954-8_30

calculations, there are many factors such as support conditions which are difficult to describe quantitatively. The increase in the depth of underground mines result in a higher demand for safety, and consequently, in-situ stress and strain monitoring. Although, in-situ stress and strain measurement has an advantage over numerical modelling methods as it can evaluate the actual stress and strain conditions of a mine, most methods can only measure the stress and strain conditions before excavation, and not throughout the whole mine development. Long-term stress monitoring is necessary to continue observing the stress and strain conditions around the excavation.

This research on the implementation of a monitoring system to detect the changes in the underground in-situ stress conditions was separated and carried out in two main components. One component is the sensor that detects the changes in the underground stress and strain conditions. The second component is the utilisation of the Ad Hoc communication (Wi-Fi Ad Hoc and Wi-Fi Direct) system to transmit the data from the sensor unit to mobile devices that then transmit data to above ground. Experiments were conducted to evaluate the functionality of the proposed technology. 


\section{Proposed Underground Rock Stress Monitoring System}

The usage of wireless sensor networks (WSNs) for underground mine monitoring systems solves problems that would usually occur when using wired communication systems, for example in the occasion of a collapse or machinery malfunction [1]. Mining companies use WSNs for locating mining workers and environmental monitoring (smoke and dust detection, the concentration of toxic gases, measurement of temperature and humidity). Figure 1 shows a collapsed section of an underground mine. As the system tries to reach equilibrium after excavation, the opening deforms and eventually collapses. A monitoring system could be employed to track these deformations that lead up to the collapse. Some examples of WSNs currently used are Wi-Fi, Bluetooth, and ZigBee [1-5]. The authors have tested the reliability of the ZigBee communication system in a Western Australian underground mine $[4,5]$.

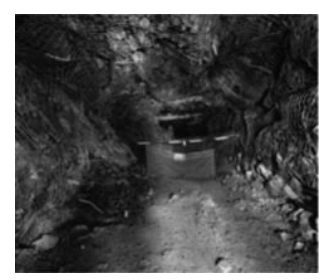

Fig. 1. Collapse of underground mine.

The underground rock stress monitoring system proposed in this research can be divided into two parts. The first part is sensing, which is the differential measurement of in-situ stress and strain. The second part of the system is the transmission of data utilising Ad Hoc communication (Wi-Fi Direct). As shown in Fig. 2, the monitoring system proposed in this research utilises Ad Hoc communication and a sensor unit that is installed in a borehole which is afterwards filled. Once blank holes are drilled, stress release occurs. Embedding the sensor units in interspersed locations will enable the detection of changes in stress applied from the rock based on the value of the strain gauge. It is also necessary to know the orientation of the sensor unit after it is embedded to detect the direction from which stress is being applied.

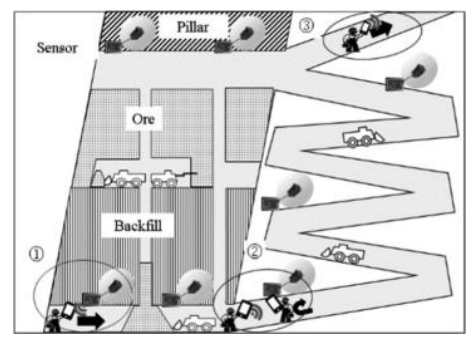

Fig. 2. System design of rock stress monitoring system.

Figure 2 shows mining workers obtaining measurement data from the sensor unit at (1). (2) shows the passing of measurement data from other sensor units and mining workers within the communication range. Measurement data is passed along from mining workers to the ground office when a worker carrying the data exits the underground mine at (3). The development of the monitoring system was regarded separately. The performance of the communication system was investigated in 3 , and the sensor unit was investigated in 4 .

\section{Ad Hoc Wireless Communication System}

Currently, communication in underground tunnels is carried out using Walkie-talkies for verbal communication, and transmission of monitoring system data is still carried out using wired communication systems (Ethernet cables, etc.). It goes without saying that, in a constantly changing environment such as an underground mine, a wireless communication system would be more suitable as there would be no need for rerouting wires. By using WSNs for computerisations in underground mines, it is possible to detect and prevent underground collapses, machinery malfunctions, aiding in the automation of mining equipment and the aggregation of information on 
various measurement instruments to create big data. WSNs can be configured as autonomous networks just by setting up a large number of nodes that can connect automatically via wireless connections. WSNs can support reliable monitoring and communication systems that can include many nodes, compared to wire communications that are very likely to fail due to cable damage.

Some examples of WSNs currently being studied include ZigBee and Wi-Fi Ad Hoc. However, although ZigBee allows many connectable terminals and multi-hop, its transmission speed is slow [3-5]. On the other hand, in addition to the advantages of WSNs, Wi-Fi Ad Hoc allows the P2P (Peer to Peer) connection between devices to be established without needing an access point, enabling the transmission of information via movement of users carrying these devices.

Communication tests on the Wi-Fi Ad Hoc system were conducted in the Osarizawa Mine in Akita Prefecture to measure the received signal strength indicator (RSSI) and transmission speed of the system [3]. The RSSI is a measurement of the power present in a received radio signal [6]. The verification of RSSI allows to test the reliability of wireless communications in a real setting. The signal reception quality is deteriorated due to wave interference. To calculate the power of the receiving antenna and consider the free-space basic propagation loss formula, Eqs. (1) and (2) are generally used:

\section{PTGtGrk}

$$
\begin{aligned}
& \text { RSSI } 1 / 420 \log \_4 p d \\
& \text { Gn } 1 / 4 \text { 4pd PAe }=k 2
\end{aligned}
$$

$$
\begin{aligned}
& \sigma^{1} p \\
& \delta^{2} p
\end{aligned}
$$

Where, RSSI is the received signal strength indicator in $\mathrm{dBm}$. PT is the transmission powers in milli-watts, respectively, $G_{t}$ and $G_{r}\left(G_{n}\right)$ are the absolute gain of the transmitting and receiving antennas in decibels relative to an isotropic radiator. $k$ and $d$ are the wavelength and distance in meters. The maximum PT of the base unit was about $1.5 \mathrm{~W} . \mathrm{A}_{\mathrm{e}}$ is the effective aperture in square meters, it is related to the physical size of the antenna. RSSI value of $-80 \mathrm{dBm}$ was defined as a reference of the minimum value for a stable connection.

Figure 3(A) shows RSSI according to distance. Figure 3(B) Throughput according to distance. It was concluded that communication is possible at a communication speed of $2.3 \mathrm{MB} / \mathrm{s}$ at a distance of up to about 110 $\mathrm{m}$ in a straight line of arched tunnels of length $2 \mathrm{~m}$ [3]. The Ad Hoc communication feature enables the proposed monitoring system to expand communication range by using the movement of passing miners. This research proposes a communication system in which mobile receivers and sensor units can communicate with each other through a relay system and transmit data from the underground to the surface (or between different points on the surface)
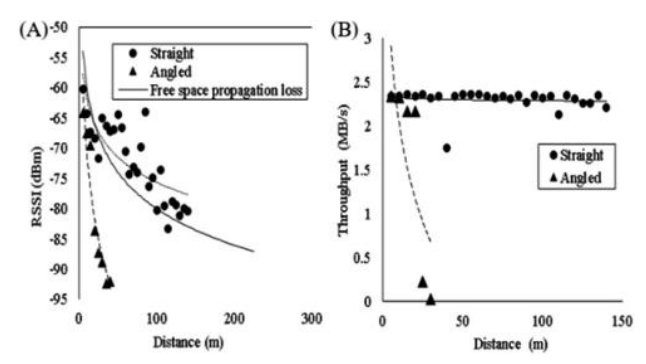

Fig. 3. Communication test results. (A) RSSI according to distance, (B) Throughput according to distance.

Throughout this research, Wi-Fi Direct, a type of Ad Hoc communication methods was utilised. Wi-Fi Direct not only has the same advantages as Wi-Fi Ad Hoc but also simplifies the complexity of the settings of an Ad Hoc system. Wi-Fi Direct estimates that similar communication performance can be obtained from using the same WiFi standard, output, and $2.4 \mathrm{GHz}$ band radio waves as Wi-Fi ad hoc.

\section{Differential Measurement of In-Situ Stress and Strain Conditions}

The following sections demonstrate techniques employed and designed for the sensing of in-situ stress; other aspects to be elaborated include stress measurement methods, the performance of the sensor unit, determination of sensor unit orientation and finally the determination of stress exerted the on the sensor unit. 


\subsection{Stress Measurement Methods}

A conventional in-situ stress measurement method is the hydraulic fracturing method in which the magnitude of stress is determined by injecting high-pressure fluids into boreholes that cause tensile failure; hence determining the principal stresses of the surrounding rock [7]. The in-situ stress measurement method requires a moderate cost efficiency as increase with depth drastically increases difficulty in implementation [7]. Another method is the overcoring method, where the physical strength of the rock can be determined by measuring the differential strain experienced by the surrounding rock when a pilot hole is over-cored and stress release occurs. However, the hydraulic fracturing method is considered to be undesirable in terms of long-term safety and stability around the tunnel after excavation because it may damage the bedrock at the measurement points.

Disadvantages of the over-coring method include the difficulty of fixing a strain gauge to the measurement point. The over-coring method also requires the elastic modulus of the surrounding rock, which at most times is oversimplified and assumed to be homogenous. Also, both the hydraulic fracturing method and the over-coring method can be used to determine the in-situ stress/strain conditions during the time of measurement, however, they cannot be used to measure the subsequent changes in the stress/strain conditions. Because the stress and strain conditions of underground mines are constantly changing as mining operations progress, in-situ stress measurement methods that can only measure in-situ stress and strain at only one point of time are insufficient. Therefore, methods of measuring differential stress after the release of stress (during excavation) are preferred. In other words, a long-term, non-destructive measurement method that can measure the difference in the in-situ stress conditions is required.

There are two methods in measuring in-situ stress, non-contact methods where the displacement of the object to be measured is measured relative to a predetermined reference space, or contact methods where sensors are directly attached to the surface of the object which its stress is to be determined. Except for situations where the vibrations of the object to be measured can be affected by the mass of the sensor, or cases when the sensor cannot be directly attached to the surface due to high temperatures, contact sensors are generally easy to use. Contact methods are also preferred because, although non-contact methods can measure displacement, they cannot measure stress conditions directly. Therefore, to determine the changes in in-situ stress that occur throughout the progress of the mine, research was focused on the development of a sensor unit that can be installed in a drill hole drilled with a support drill bit ( $\mathrm{u} 60 \mathrm{~mm}$ ).

The sensor unit developed was a solid rectangular box made of a stainless-steel plate. In addition to the strain gauges attached to the inside of the sensor on the XYZ plane, a 9-axis sensor (LSM9DS1) that included an accelerometer, gyroscope and a magnetometer to determine the orientation of the sensor after its installation was also fixed in the sensor unit. The obtained data is transmitted to the data logger through a built-in wire cable.

\subsection{Determining Sensor Unit Orientation}

Figure 4(A) shows a sensor unit affixed with a portable Ad Hoc communication terminal. The purpose of the 9axis sensor (LSM9DS1) is to determine the orientation of the sensor after it has been installed, by calculating the acceleration (accelerometer), change in angular velocity (gyroscope) and the direction of true north (magnetometer). The sensor fusion of these three sensors compensate for the disadvantages of the respective sensors and improve the accuracy when determining the orientation of the sensor after installation.
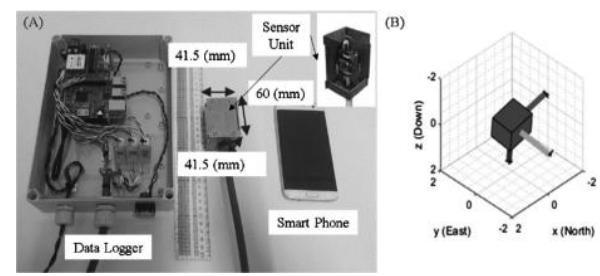

Fig. 4. Sensing equipment and orientation calculation results. (A) Sensor unit and mobile device, (B) Orientation of sensor unit.

In this research, a software was used to determine the orientation of the sensor unit. Figure 4(B) illustrates the actual orientation of the sensor, confirming that the real-time orientation of the sensor can be determined accurately. Data from the readings of the stress measurements by the strain gauges attached to the inside of the sensor surfaces are combined with the sensor orientation to determine both the direction and magnitude of stress applied on the 
sensor. Figure 5(A-C) shows $\mathrm{X}$ and $\mathrm{Y}, \mathrm{Z}$ axis reliability with linearity error of less than $\pm 0.1 \%$. Figure 5 (D) is a disassembly view of the unit housing.
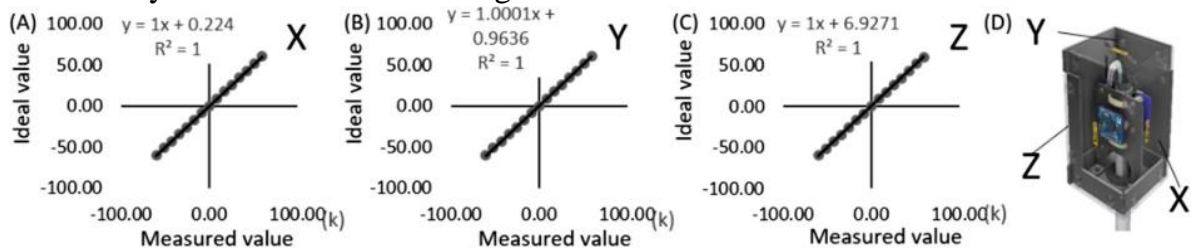

Fig. 5. Sensing unit reliability. (A) $\mathrm{X}$ axis reliability, (B) $\mathrm{Y}$ axis reliability, (C) $\mathrm{Z}$ axis reliability,

(D) Sensor unit disassembly view

\subsection{Determination of Stress Exerted on Sensor Unit}

A uniaxial compression test was conducted on the sensor unit, and the stress (in-situ stress) applied to the sensor unit is determined from the load and displacement curve obtained. The relationship between stress and strain was determined from the value of the strain gauge. The in-situ stress conditions of a mine-site can be determined from the strain of the sensor unit embedded in the borehole.

During this research, indoor experiments were conducted as basic experiments. A manual uniaxial compression tester (with a capacity of $200 \mathrm{~N}$ ) was used to conduct a load response test. The load exerted on the sensor unit was increased with increments of $0.5 \mathrm{~N}$, and the readings from the strain gauge were used to form a load-displacement curve (which shows elastic deformation) and data such as the acceleration, angular velocity and the direction of true north (based on geomagnetism) were obtained from the 9-axial sensor. The data acquired at a sampling frequency of $1 \mathrm{~Hz}$ was transmitted from the sensor unit data logger to a mobile device using the Ad Hoc communication (Wi-Fi Direct) mentioned above. The frequency of the communication between the mobile device and data logger can be controlled by using the developed application. The loaddisplacement curve is shown in Fig. 6(A), where the line is plotted linearly. The Fig. 6 (B) shows the displacement-strain curve, where the X's line is plotted linearly.
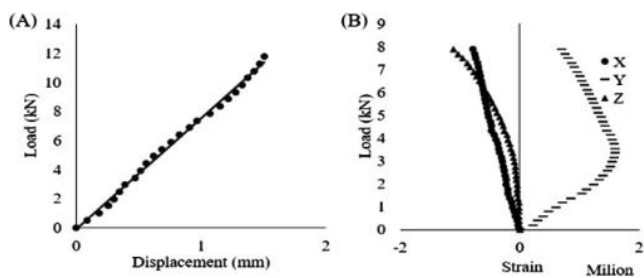

Fig. 6. Uniaxial compression test results. (A) Load-displacement curve, (B) Displacement-strain curve.

Up to $5 \mathrm{MPa}$ of load was applied to the sensor unit during the load response test. The data from the 9-axis sensor and the 3-axis strain gauges change with time. Based on the values from the strain gauge, it is possible to measure the in-situ stresses in underground mines. In order to determine the in-situ stress and displacement that occurs in underground mines, it is important to not only be able to detect the changes in the magnitude of in-situ stress but to also know the orientation of the sensor to determine the direction that the force is being applied from.

The total amount of data transmitted from the sensor is about $13 \mathrm{MB} / \mathrm{day}$, which is fairly small compared to the amount of data that can be transmitted by using the proposed communication system. The results of these tests verify the feasibility of the proposed monitoring system.

\section{Conclusion}

The implementation of better communication systems will improve the productivity and safety of underground mines. This research proposed a monitoring system that was separated into two components, sensing and data transmission. Among the several WSNs available, this research focused on Wi-Fi Direct is considered to be suitable for informatisation in underground mines. Communication tests were carried out to investigate the performance of the proposed communication system. By measuring the RSSI and the transmission speed, the distance and transmission speed that data can be stably transmitted were determined. As a result of the conducted 
communication tests, it was determined that during stable wireless communication (at an RSSI of more than -80 $\mathrm{dBm}$ ), it is possible to communicate at a transmission speed of $2.3 \mathrm{MB} / \mathrm{s}$ at a point $95 \mathrm{~m}$ away from the data logger in a straight line.

The efficient measurement and understanding of stress distribution underground are important in the safe design of underground mines. Although, it is possible to determine the in-situ stress/strain measurement beforehand, it is important to be able to monitor the long-term changes in the stress and strain conditions. This research proposed sensing the in-situ stress conditions based on the rock stress changes resulting from mine development. Sensor fusion of a 9-axial sensor installed inside the sensor unit is used to determine the orientation of the sensor unit post-installation, while the stress exerted onto the sensor unit can be measured using the strain gauges attached on the inside of the sensor unit. By combining these results, the magnitude and direction of the in-situ stress can be determined. The amount of data regarding in-situ stress totals to about $13 \mathrm{MB} / \mathrm{day}$, which is relatively small compared to the amount of data that can be transmitted by the proposed communication system. Based on the results of the tests carried out, the functionality and feasibility of the proposed monitoring system have been verified.

\section{References}

1. Moridi, M.A., Kawamura, Y., Sharifzadeh, M., Chanda, E.K., Jang, H.: An investigation ofunderground monitoring and communication system based on radio waves attenuation using ZigBee. Tunn. Undergr. Space Technol. 43, 362-369 (2014)

2. Kawamura, Y., Dewan, A.M., Veenendaal, B., Hayashi, M., Shibuya, T., Kitahara, I.,Nobuhara, H., Ishii, K.: Using GIS to develop a mobile communications network for disasterdamaged areas. Int. J. Digit. Earth 7, 279-293 (2014)

3. Ikeda, H., Kawamura, Y., Tungol, Z.P.L., Ito, Y., Jang, H.: Development of undergroundspace communication systems using Wi-Fi Ad Hoc for smart mining. Jpn. Soc. Geoinf. 29, 3- 11 (2018)

4. Moridi, M.A., Kawamura, Y., Sharifzadeh, M., Chanda, E.K., Wanger, M., Okawa, H.: Performance analysis of ZigBee network topologies for underground space monitoring and communication systems. Tunn. Undergr. Space Technol. 71, 201-209 (2018)

5. Moridi, M.A., Sharifzadeh, M., Kawamura, Y., Jang, H.: Development of wireless sensornetworks for underground communication and monitoring systems (the cases of underground mine environments). Tunn. Undergr. Space Technol. 73, 127-138 (2018)

6. Sun, H.Y., Bi, L.J., Lu, X., Guo, Y.J., Xiong, N.X.: Wi-Fi network-based fingerprinting algorithm for localization in coal mine tunnel. J. Internet Technol. 99, 1-10 (2015)

7. Funato, A., Ito, T.: A new method of diametrical core deformation analysis for in-situ stressmeasurements. Int. J. Rock Mech. Min. Sci. 91, 112-118 (2017) 